\title{
IMPROVING THE INTEROPERABILITY OF DISASTER MODELS: A CASE STUDY OF PROPOSING FIREML FOR FOREST FIRE MODEL
}

\author{
Wenyu Jiang ${ }^{1}$, Fei Wang ${ }^{2}$ *, Qingxiang Meng ${ }^{1}, *$, Zhanghua $\mathrm{Li}^{2}$, Binbin Liu ${ }^{2}$, Xiaocui Zheng ${ }^{2}$ \\ ${ }^{1}$ School of Remote Sensing and Information Engineering, Wuhan University, China - (jiangone, mqx)@whu.edu.cn \\ ${ }^{2}$ Graduate School at Shenzhen, Tsinghua University, China - (Wang.fei@sz, zh-li15@mails, liubb16@mails, \\ Zheng.xiaocui@sz).tsinghua.edu.cn
}

Commission III, ICWG III/IVa

KEY WORDS: FireML, Forest Fire Model, 3DGIS Disaster Management System

\begin{abstract}
:
This paper presents a new standardized data format named Fire Markup Language (FireML), extended by the Geography Markup Language (GML) of OGC, to elaborate upon the fire hazard model. The proposed FireML is able to standardize the input and output documents of a fire model for effectively communicating with different disaster management systems to ensure a good interoperability. To demonstrate the usage of FireML and testify its feasibility, an adopted forest fire spread model being compatible with FireML is described. And a 3DGIS disaster management system is developed to simulate the dynamic procedure of forest fire spread with the defined FireML documents. The proposed approach will enlighten ones who work on other disaster models' standardization work.
\end{abstract}

\section{INTRODUCTION}

Fire disaster is widely regarded as one of the most destructive hazards, which has caused a huge loss of life and property. With the rapid development of social urbanization process, the frequency of fire accident is increasing year by year, which seriously affects human's normal production and life. Fire security gradually grows into one of the most concerned events of the public. For instance, in January 2014, a fire broke out in Shangri-La County of Yunnan province, and destroyed 343 historic blocks and finally resulted in a direct economic loss of nearly 90 million RMB (Wang, 2016a). In October 2017, the biggest forest fire in American history took place in California, which has caused at least 42 deaths, 7000 blocks damage and urgently evacuated almost 100,000 inhabitants. Countless instances illustrate the severity of fire disaster, thus it is significantly necessary to carry out deeper researches for preventing and controlling the fire disaster.

In recent years, a large number of researches on fire disaster has been done on the fields like detection, forecast, simulation, rescue and visualization. Izumi et al. (2012b) proposed a new technique for visualization of forest fire smoke plumes using MODIS data, Li et al. (2017b) proposed GIS based dynamic modelling of fire spread with cellular automation model, $\mathrm{P}$. Vijay et al. (2015b) showed us a GIS fire rescue system using small airborne vehicles - Quad copter, Fei et al. (2005a) gave a three-dimensional visualization model of fire frame using particle motion animation. In general, researches on the mechanism and visualization of fire model are relatively mature, and their achievements play an important role in practical firefighting applications. Many advanced fire disaster management systems are equipped with comprehensive functions such as fire forecast, fire simulation and visualization, fire analysis, fire rescue decision support and so on. However, most of these systems have their own proprietary data structures, especially for the input and output data of the fire model. Thus, it results in a bad compatibility and hard data interoperability when communicating with other disaster management systems. During the disaster management procedures, and particularly for emergency response, related information must be exchanged smoothly among different disaster management systems or subsystems in order to provide timely analyzing results for the decision makers. Moreover, a disaster may not be a standalone disaster and may cause other disaster types, which is studied as the disaster chain (Zheng et al, 2017b). For example, a forest fire may cause explosions and the chemical leakages may happen if there are nearby chemical factory. Therefore, it is very important to have standardized data specifications for disaster models, which can ensure the interoperability of those disaster models when applying in disaster chains or different information systems.

GML (Geography Markup Language) is a type of generalized markup language proposed by OGC (Open Geospatial Consortium), which is aimed at providing efficient transmission and storage encoding for geospatial information (Yue, 2011). GML has the capability to solve the problem of exchanging and sharing geospatial data between different systems and can provide the spatial information coding method adapted to the Internet environment. In addition, a variety of spatial and nonspatial data is supported by GML. Considering the advantages of GML, it has been widely applied in many spatial applications including storage, parsing, querying and visualization, as well as the use of GML for mobile devices and web services (ChanTien et al, 2007a). There is no doubt that the disaster science also has the demand for a standardized markup language.

In this paper, we take the standardization work of forest fire model as a study case and propose a standard generalized fire 
model language FireML to standardize the input and output parameters of forest fire model with aim of providing the possibility for efficient interoperability with other information system. Meanwhile, to testify the feasibility of the defined FireML, a 3DGIS disaster management is developed to demonstrate the usage of FireML and reconstruct the dynamic procedure of forest fire spread.

Our paper is organized as follows. In Section 2, the forest fire model is described. Then considering the input and output parameters of this model, the structure and composition of FireML is presented. In Section 3, the implemented 3D-GIS disaster management system is introduced and the simulation experiment is constructed with the defined FireML. Section 4 shows the discussions and concludes the paper.

\section{THE STRUCTURE AND COMPOSITION OF FIREML}

FireML is specially designed for describing the forest fire model in a standardized way and aimed at providing an efficient interaction platform for diverse disaster models. Given that the disaster chain, different disaster models need to interact with each other. However, most models are distributed in different place and model interactions always use the Internet to exchange data. Therefore, a type of standard generalized markup language which can exchange and share data between different disaster models and provide the disaster information coding method adapted to the Internet environment is urgently needed.

In our paper, we extend GML to define the concept of FireML. And the proposed FireML inherits the good structure characteristics of GML. It strictly uses coupled tags to express the value of fire model data and do not allow chaotic nesting of tags for the sake of easily parsing the FireML documents. Tags strategy has an advantage in format specification but fails to constrain the value filled between tags. In order to make the value meet the demand of easily parsing and efficiently interacting, we adopt the schema models to verify the validity of the tag value and standardize the logical structure of fireML documents. In order to design the FireML more properly and practically, we take the forest fire model as a study case and standardize the input and output document of the adopted model.

\subsection{Forest Fire Model}

The model of fire spread is the core of fire management system. In recent years, dozens of fire spread models have been proposed. Those models can be summarized into three categories: namely empirical models, probability models and physical models (Zhao et al, 2006a). Empirical models are mainly based on the statistical analysis of filed data and experimentally obtained data. Probability models adopt mathematical equations to describe the stochastic process of the fire spread and match the indefinite nature of fire spreading (Zhao et al, 2003a). While Physical models are based on the fire spread rules, including the physical substances and energy laws. In our system, we adopt an empirical forest fire model (Wang, 1983a; Wang, 1992a; Mao, 1993a). Firstly, an initial fire spread speed $\mathrm{R}_{0}(\mathrm{~m} / \mathrm{min})$ is calculated by empirical formula as follows:

$$
R_{0}=a * T+b * V+c * H-D
$$

where $\quad \mathrm{T}=$ air temperature

$\mathrm{V}=$ average wind level in the mid-afternoon

$\mathrm{H}=$ the minimal humidity value of a day

$\mathrm{a}, \mathrm{b}, \mathrm{c}, \mathrm{D}=$ constant coefficient $(\mathrm{a}=0.03, \mathrm{~b}=0.05$, $\mathrm{c}=0.01, \mathrm{D}=0.3$ )

Considering the influence of wind and terrain, the speed of fire spread $\mathrm{R}(\mathrm{m} / \mathrm{min})$ is determined by formulas below:

$$
R=R_{0} * K_{S} * e^{3.533 *(\tan \theta)^{1.2}} * e^{0.1783 * V}
$$

where $\mathrm{K}_{\mathrm{s}}=$ coefficient of fuel configuration pattern

$\theta=$ the angle of terrain gradient

$\mathrm{V}=$ average wind level in the mid-afternoon

Then the Huyghens principle, widely applied to present the area of object spread by continuous expanding polygons, is then adopted to fit the dynamic process of fire spread (Knignt et al, 1993a). Firstly, starting with the ignition point, we calculate the fire speeds of eight directions (East, Southeast, South, Southwest, West, Northwest, North, Northeast) by the empirical formulas. Secondly, the spread distance of each unit time is calculated and the coordinate points from eight directions will be updated. Finally, With interpolating and fitting these coordinate points, the accurate scope of fire spread at any certain time can be obtained. The dynamic process of fire spread is as Figure 1 shows.

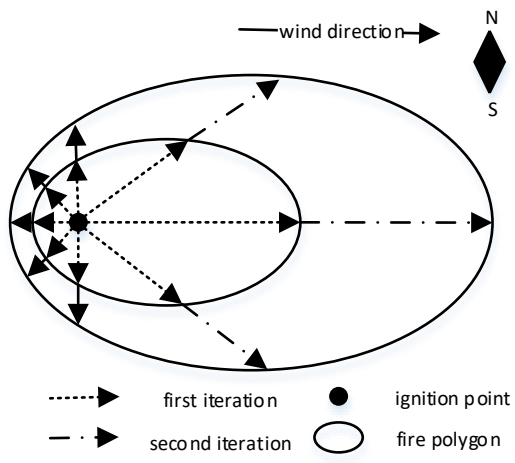

Figure 1. The dynamic process of fire spread

\subsection{The Input FireML Document}

Based on the forest fire model, the input FireML document should contain these indispensable elements: ignition coordinates, time parameter, environment factors and other reference information as described in Table 1.

\begin{tabular}{|c|c|}
\hline Elements Names & Child elements names \\
\hline fml:simpleMulPoint & fml:posList \\
\hline \multirow{3}{*}{ fml:timeParameter } & fml:beginTime \\
\cline { 2 - 2 } & fml:TimeLength \\
\cline { 2 - 2 } & fml:TimeInterval \\
\hline \multirow{4}{*}{ fml:environment } & fml:humidity \\
\cline { 2 - 2 } & fml:windSpeed \\
\cline { 2 - 2 } & fml:temperature \\
\cline { 2 - 2 } & fml:windDirection \\
\hline
\end{tabular}

Table 1. The main elements of input document 
The element of fml:simpleMulPoint describes the ignition points with a default coordinate system of EPSG:4326. And its child element fml:posList consists of a collection of latitude and longitude in sequence to state the accuracy coordinates of ignition points. The element of fml:timeParameter includes the beginning time, time length and time interval for fire model. While the element of fml:environment provides the indispensable environment factors such as wind speed, wind direction, temperature and humidity. Those environment parameters play a key role in simulating the forest fire spread. In addition to the three main elements, many other attachments, like description and mete data property, are also optional in the input FireML document.

\subsection{The Output FireML Document}

After receiving the input FireML document, the forest fire model will parse the document, extract all model parameters and then simulates the fire spreading process and encapsulates the outcome into the output document. The elements of the output FireML document are shown in Table 2.

\begin{tabular}{|c|c|}
\hline Elements Names & Child elements names \\
\hline \multirow{2}{*}{ fml: boundedBy } & fml:lowerCorner \\
\hline & fml: upperCorner \\
\hline \multirow{7}{*}{$\begin{array}{c}\mathrm{fml}: \\
\text { firePolygonCollection }\end{array}$} & fml: timePosition \\
\hline & fml: fireLength \\
\hline & fml: fireArea \\
\hline & damageLevel \\
\hline & personnelLoss \\
\hline & economicLoss \\
\hline & simplePolygon \\
\hline \multirow{4}{*}{ fml:environment } & fml:humidity \\
\hline & fml:windSpeed \\
\hline & fml:temperature \\
\hline & fml:windDirection \\
\hline \multirow{2}{*}{ fml:modelParameter } & fml:modelName \\
\hline & fml:modelSetting \\
\hline fml:simpleMulPoint & fml:posList \\
\hline \multirow{3}{*}{ fml:timeParameter } & fml:beginTime \\
\hline & fml:TimeLength \\
\hline & fml:TimeInterval \\
\hline
\end{tabular}

Table 2. The main elements of output document

The elements of fml:simpleMulPoint, fml:environment and fml:timeParameter are the same as the ones in the input documents. The element of fml:boundedBy uses the child elements fml:lowerCorner and fml: upperCorner to present the boundary of the biggest fire spread polygon within the limited simulation time. To describe the fire spread polygon more detailedly, the element of fml:firePolygonCollection is used to present the sequential spatial data and non-spatial attribute data generated by the forest fire model. The granularity of fire simulation is controlled by the elements of time length and time interval in the input FireML document. And a series of fire polygon is used to fit the dynamic process of fire spread. For each certain fire polygon, we adopt the element of $\mathrm{fml}$ :firePolygon to represent the geographic coordinates and the attribute data of the fire spread polygon. Here, the geographic coordinates are presented by the element of fml:simplePolygon, which consists of a collection of latitude and longitude in sequence. As for the attribute data, the element of fml: timePosition is designed for the accurate time towards the fire polygon like 2017-11-11T16:00:00Z. And the elements of fml:fireLength and fml:fireArea are adopted to quantitatively explain the perimeter and area of fire polygon. In particular, to express the estimated loss caused by the fire, we propose the element of fml:damageLevel to describe the fire severity at four different degrees: particularly serious accident, major accident, serious accident and normal accident (State Administration of Work Safety, 2015). The fire severity is determined by the loss of life and economics represented by the elements of fml:personnelLoss and fml:economicLoss. Those standardized elements for forest fire model not only contribute to improve the interoperability of various disaster models, but also provide decision support of disaster rescue for the government.

\subsection{FireML XSD Document}

XSD is the XML Schema Definition used for constructing the legal XML document. Based on the XSD document, defining the data facets and data model for verifying the correctness of FireML documents can be easy.

\begin{tabular}{|c|c|c|}
\hline Elements & Data Type & Example \\
\hline fml: timePosition & xs:datetime & $\begin{array}{c}\text { 2017-11-15T } \\
16: 00: 00 Z\end{array}$ \\
\hline fml: fireLength & xs:decimal & 300.00 (meter) \\
\hline fml: fireArea & xs:decimal & 400.00 (meter) \\
\hline fml:damageLevel & xs: integer & 1 \\
\hline fml:personnelLoss & xs:integer & 30 \\
\hline fml:economicLosses & xs:decimal & 30(million) \\
\hline fml:humidity & xs:decimal & 80 \\
\hline fml:windSpeed & xs:decimal & $5.0(\mathrm{~m} / \mathrm{s})$ \\
\hline fml:temperature & xs:decimal & $26^{\circ} \mathrm{C}$ \\
\hline fml:windDirection & xs:string & $\mathrm{N}$ \\
\hline fml:modelName & xs:string & forest model \\
\hline modelSpecification & xs:string & none \\
\hline fml:beginTime & xs:datetime & $\begin{array}{c}\text { 2017-11-15T } \\
16: 00: 00 \mathrm{Z}\end{array}$ \\
\hline fml:TimeLength & xs:integer & 1(hour) \\
\hline fml:TimeInterval & xs:integer & 0.5 (hour) \\
\hline
\end{tabular}

Table 3. The data type and example of elements

In our paper, we design the XSD documents for FireML extended by the ones of GML. Most of GML XSD documents are inherited by FireML XSD documents such as gml.xsd, feature.xsd, coorinateSystems.xsd, geometryBasic2d.xsd, defaultStyle.xsd, temporal.xsd and topology.xsd. Meanwhile, an 
appropriative XSD document called fireInformation.xsd for forest fire model data is proposed to constrain the element value in FireML documents. In the fireInformation.xsd document, the elements, element structure and element attributes are described in detail. At same time, the data type and data constraint or default value are defined clearly. The detailed data type and examples of elements for the FireML documents in the XSD document are shown in Table 3.

In Table 3, the element of fml:damageLevel is represented by an index value such as 1 to 4 to show the degree of fire damage; the element of fml:humidity adopts a centesimal system to present the environment humidity whose value ranges from 0 to 100. And the element of fml:windSpeed takes meter per minute as unit and its value ranges from 0 to 62 ; while the element of fml:temperature uses degrees centigrade to present the air temperature with a value ranging from -10 to 40 ; and the fml:windDirection contains eight values to express the wind direction like East(E), Southeast(SE), South(S), Southwest(SW), West(W), Northwest(NW), North(N), Northeast(NE).

\section{THE 3DGIS DISASTER MANAGEMENT SYSTEM}

With the development of 3D visualization and virtual reality technologies, the 3DGIS system becomes increasingly popular in simulating physical geography appearance and making spatial analysis and statistics. Therefore, many researchers employ the 3DGIS system to model, visualize and analyze diverse disaster types in the disaster management fields, such as the fire modelling work (Huang et al, 2012b).

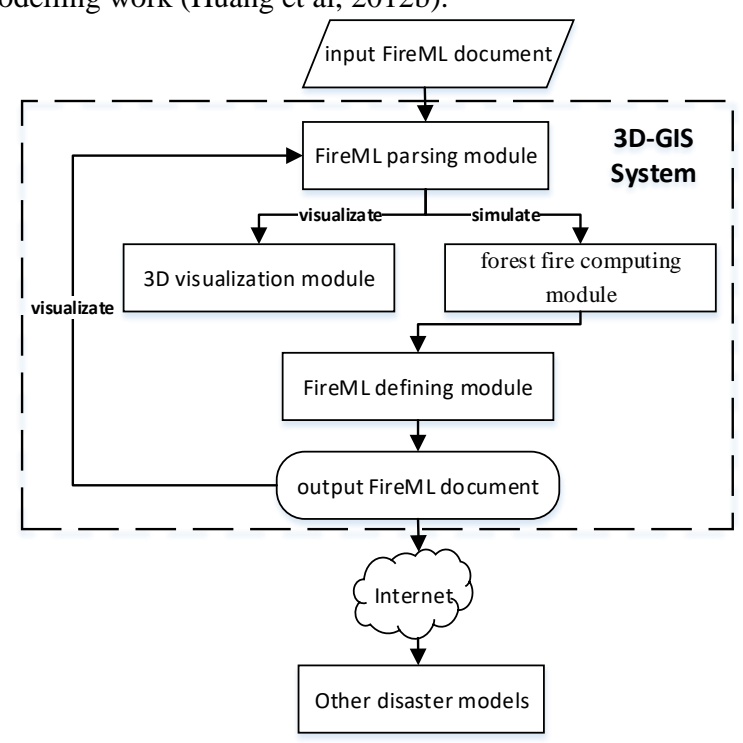

Figure 2. The simulation process with the defined FireML in the 3DGIS disaster management system

In our research, in order to testify the feasibility of the defined FireML, a 3DGIS disaster management system is developed to demonstrate the usage of FireML and reconstruct the procedure of forest fire spread. This system contains four core functional modules: FireML defining module, FireML parsing module, forest fire computing module, 3D visualization module. With the support of system functional modules, the process of fire simulation with the defined FireML mainly contains four steps. Firstly, users import the input FireML document into the system. Secondly, the FireML parsing module of the system begins to parse the parameters of forest fire model from the input document by the FireML standard and send them to forest fire computing module. Thirdly, the forest fire computing module simulates the fire spread process and invokes the FireML defining module to encapsulate the simulation outcome into the output FireML document. Finally, the output FireML document can be parsed to visualize the dynamic process of fire spread by the 3D visualization module and sent to other disaster model as an input document via the Internet. The simulation process of the 3DGIS disaster management system for forest fire spread is shown in Figure 2.

In the Figure 2, FireML defining and parsing modules are designed by the FireML XSD documents, while the forest fire computing module is determined by the adopted forest fire model. And the $3 \mathrm{D}$ visualization module is constructed by the $3 \mathrm{D}$ virtual geographic scene.

\subsection{Construction of the 3D Virtual Geographic Scene}

The aim of constructing the 3D virtual geographic scene is to visualize the process of forest fire spread in a more vivid way and provide an immersive feeling of fire disaster for the rescue decision makers. The virtual geographic scene in our paper is made up of some fundamental data like the digital elevation model (DEM), 3D building models, remote sensing images and environment elements. The core component of 3D sense is the DEM, which contains the terrain information and takes a good compatibility with remote sensing data and other GIS raster layer. With all initial data transforming their geographic coordinates into the same map projection system, the basic 3D geographic scene can be constructed by overlaying the remote sensing images on DEM. And the overlaying function is supported by many software such as Skyline, ArcGIS and so on. Then 3D building models and environment elements are added into the basic scene for a more realistic feeling. Meanwhile, a particle system, widely used for the irregular and fuzzy objects and phenomena (William et al, 1998a), is adopted to simulate and visualize the fire in a more vivid effect.

\subsection{Experiment with the Defined FireML}

To testify the feasibility of the defined FireML, an experiment is done by our 3D disaster management system in the Tanglang mountain nearby the University Town of Shenzhen in Guangdong province. As for the operation of the system for the usage of the FireML documents, first we click the import button to import the input FireML document. Then we click the beginning button to calculate the simulating outcome. Next the outcome will be exported into an output FireML document after clicking the export button. Finally, the dynamic process of fire spread will be shown in the 3D virtual geographic scene if we click the dynamic presentation button. As we shown in Figure 3 .

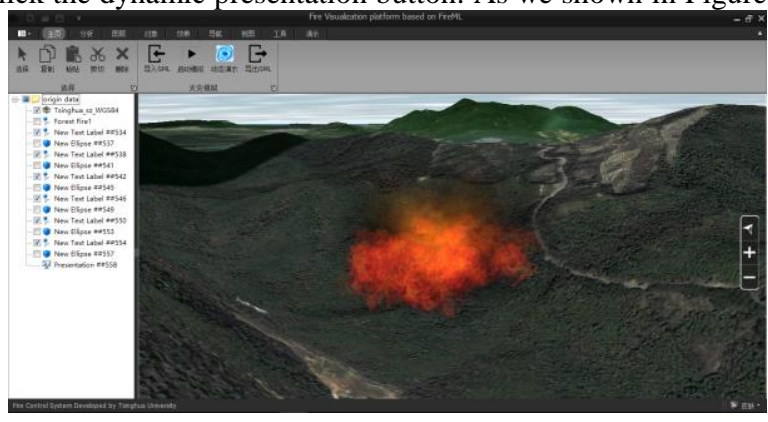

Figure 3. The dynamic process of fire spread in 3D scene 


\section{DISCUSSION AND CONCLUSION}

In our work, we propose a novel data format FireML to standardize the input and output parameters of forest fire model with the aim of improving the interoperability of disaster models. The elements of FireML documents are designed by the parameter requirements of empirical forest fire model. To testify the feasibility of the defined FireML, a 3DGIS disaster management system is developed to demonstrate the usage of FireML and reconstruct the procedure of forest fire spread. Using this system, a simulation experiment is implemented by a forest fire in the Tanglang Mountain and the result of our experiment shows that FireML applied in the 3DGIS disaster management system has a good interoperability and great potential. Although only a forest fire model is adopted to demonstrate the standardization work, the FireML shows its good extendibility to apply to other types of fire models, even other disaster models. As the research of disaster science moves along, we find that the problems of messy various disaster data format and the difficulty in efficient interaction between disaster models are increasingly apparent. There comes an urgent demand of standardized data specifications for disaster models. Those specifications, like FireML, have the capacity to improve the interoperability of disaster models, even promote the disaster science into a new level.

In the future, more various elements related to the forest fire model will be considered being concluded into FireML documents such as the firefighting element for resource allocation and decision support. Meanwhile, FireML will extend to adapt other typical fire models to provide more flexible model options. Further, based on the theory of disaster chain, the researches in Disaster Markup Language for providing a unified standard data format for all disaster models is indeed worthy of in-depth study.

\section{ACKNOWLEDGEMENTS}

This paper is supported by National Key Research and Development Program of China (Grant No. 2016YFC0803107 and Grant No. 2016YFB052601) and Shenzhen Technology Innovation Program (Basic Research JCYJ20170307152553273).

\section{REFERENCES}

Wang, Y., 2016a. The Moonlight City of born of fire - ShangriLa fire in Yunnan province in 2014. Disaster Reduction in China, 1 (22), pp. 24-27.

Izumi, N., Jun-ichi, K., Koichi, K., 2012b. A new technique for visualization of forest fire smoke plumes using MODIS data. 2012 IEEE International Geoscience and Remote Sensing Symposium (IGARSS), Munich, Germany.

Li, $\quad$ Z., Wang, F., Zheng, X., Jiang, W., Meng, Q., Liu, B., 2017b. GIS based Dynamic Modelling of Fire Spread with Heterogeneous Cellular Automation Model and Standardized Emergency Management Protocol. The 3rd ACM SIGSPATIAL International Workshop on the Use of GIS in Emergency Management (EM-GIS 2017), California, USA.

P.Vijay, K., Akhil, C., Jammi, A., G. Lakshmi, N., 2015b. GIS based Fire Rescue System for Industries using Quad copter - A Novel Approach. International Conference on Microwave, Optical and Communication Engineering, Bhubaneswar, India.
Fei, S., Peng, Y., Lu, G., Liu, Z., 2005a. Visualization of turbulent Combustion flame based on particle System. Journal of Computer Aided Design \& Computer Graphics, 17(3), pp. 461-466.

Zheng, L., Wang, F., Zheng, X., Liu, B., 2017b. A Distinct Approach for Discovering the Relationship of Disasters using Big Scholar Datasets. 5th Annual International Conference on Geo-Spatial Knowledge and Intelligence, Chiang Mai, Thailand.

Yue, P., 2011. Web Geographic Information Systems and Services.

Chang-Tien, L., Raimundo, F., Lakshmi, N., Kou, Y., 2007a. Advances in GML for Geospatial Applications. Geoinformatica, 11(1), pp. 131-157.

Zhao, S., Xiong, L., Ren, A., 2006a. The GIS-based Simulation of Urban Mass Fire Spread. Fire Safety Science, 15(3), pp. 128137.

Zhao, Z., Yu, S., Zhong, J., 2003a. Probability model for hazard analysis of post-earthquake fire occurrence and spread among buildings. Earthquake Engineering and Engineering Vibration, 23(4), pp. 183-187.

Wang, Z., 1983a. The measurement method of the wildfire initial spread rate. Journal of Mountain Research, 1(2), pp. 4251 .

Wang, Z., 1992a. Current forest fire danger rating system. Journal of natural disaster, 1(3), pp. 39-44.

Mao, X., 1993a. The Influence of Wind and Relief on the Speed of the Forest Fire Spreading. Quarterly journal of applied meteorology, 1(4), pp. 100-104.

Knight, I., Coleman, J., 1993a. A Fire Perimeter Expansion Algorithm-Based on Huygens Wavelet Propagation. International Journal of Wildland Fire, 3(2), pp. 73-84.

State Administration of Work Safety., 2015. The Byelaw governing reporting, investigation and handling of production safety accidents.

http://www.chinasafety.gov.cn/newpage/Contents/Channel_206 97/2015/0420/258349/content_258349.htm

Huang, H., Tang, L., Li J., Chen, C., 2012b. Simulation and visualization of forest fire growth in an integrated 3D virtual geographical environment - a preliminary study. 2012 20th International Conference on Geoinformatics, Hong Kong, China.

William, T., 1998a. Particle Systems - a Technique for Modelling a Class of Fuzzy Objects. Association for Computing Machinery, 2(2), pp. 91-108. 